\title{
Perivascular Epithelioid Cell Tumor Arising from Ciliary Body Treated by Local Resection
}

\author{
Hiroshi Goto ${ }^{a}$ Yoshihiko Usui ${ }^{a}$ Toshitaka Nagao ${ }^{b}$ \\ Departments of a Ophthalmology and ${ }^{b}$ Anatomic Pathology, Tokyo Medical University, \\ Tokyo, Japan
}

\section{Key Words}

Perivascular epithelioid cell tumor · Ciliary body $\cdot$ Local resection

\begin{abstract}
Aims: Perivascular epithelioid cell tumor (PEComa) is a mesenchymal neoplasm originating from perivascular myoid cells. We report a case of PEComa arising from the ciliary body. Methods: Case report. Results: A 13-year-old girl was referred to our department with a clinical diagnosis of ciliary body melanoma in her right eye. Her visual acuity was 20/600 OD. Slit-lamp examination revealed a brown tumor behind the iris. The ocular fundus could not be observed due to a cataract. Ultrasonography depicted an oval mass approximately $10 \mathrm{~mm}$ in diameter at the ciliary body. The tumor was successfully treated by local resection, and the patient's visual acuity improved to 20/20. Histopathological and immunohistochemical findings of the excised tumor were compatible with the diagnosis of PEComa of the ciliary body. No local recurrence of the tumor was observed for over 4 years after surgery. Conclusion: A very rare case of PEComa of the ciliary body was successfully treated by local resection, with favorable visual outcome and no recurrence for several years. PEComa can be differentiated from other ciliary body tumors by immunohistochemical study.

(C) 2014 S. Karger AG, Basel
\end{abstract}

\section{Introduction}

Perivascular epithelioid cell tumors (PEComas) are mesenchymal neoplasms originating from perivascular myoid cells [1]. According to the review by Hornick and Fletcher [2], PEComa most commonly involves the retroperitoneal, visceral, abdominal and pelvic sites. 
Ocular Oncology

and Pathology

Goto et al.: Perivascular Epithelioid Cell Tumor Arising from Ciliary Body Treated by Local Resection
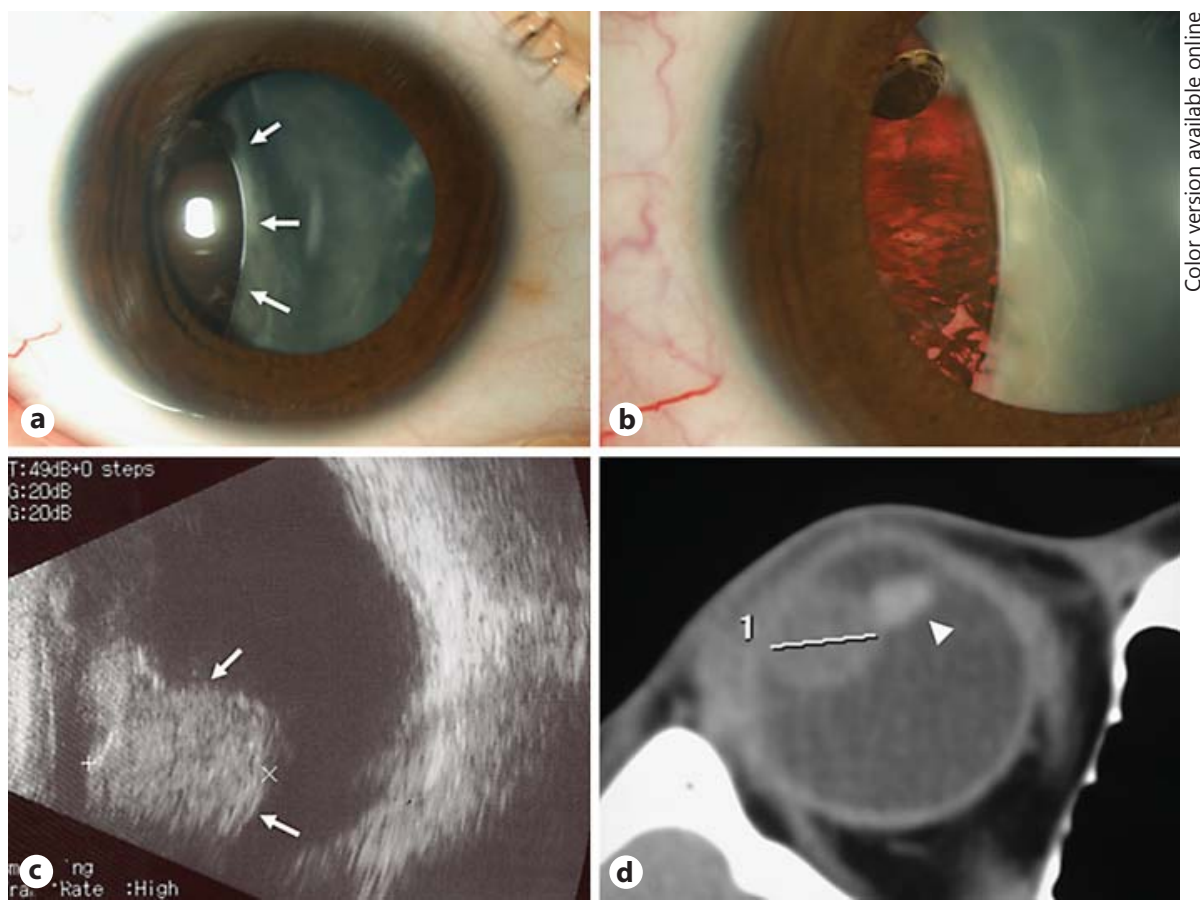

Fig. 1. Clinical features of the tumor. a Slit-lamp photograph showing a brown tumor (arrows) behind the iris and cataract. $\mathbf{b}$ The temporal episcleral vessels were slightly dilated. Note the presence of a cataract with indentation of the lens caused by the tumor. c Ultrasonography demonstrated an oval and solid mass (arrows) at the ciliary body. $\mathbf{d}$ Axial computed tomography showed an oval tumor $11 \mathrm{~mm}$ in diameter at the ciliary body. Note the deformed and tilted lens caused by the tumor (arrowhead).

PEComa can arise in unusual sites including the head and neck, but most of these cases were reported as small case series. Only 4 cases of PEComa have been reported in the ophthalmic literature [3-5]. Furusato et al. [5] described a case of intraocular PEComa, but did not provide sufficient clinical data and outcome after treatment.

We report a case of PEComa arising from the ciliary body, which was successfully treated by local resection of the tumor and achieved favorable visual outcome.

\section{Case Report}

A 13-year-old girl was referred to the Department of Ophthalmology, Tokyo Medical University Hospital on May 28, 2009 with a clinical diagnosis of ciliary body melanoma in her right eye. Her best corrected visual acuity was 20/600 OD and 25/20 OS. Moderate exotropia was present. Slit-lamp examination revealed a brown tumor behind the iris from 7 to 11 o'clock in her right eye (fig. 1a, b). The temporal episcleral vessels were slightly dilated. The ocular fundus could not be observed due to a cataract. Ultrasonography depicted a sharply defined, oval and solid mass approximately $10 \mathrm{~mm}$ in diameter (fig. 1c). Computed tomography (axial view) revealed an oval tumor measuring $10 \times 11 \mathrm{~mm}$ at the ciliary body, and a deformed lens compressed by the tumor (fig. 1d). MR examination of the tumor showed hyperintensity on the T1-weighted image and hypointensity on the T2-weighted image. Single photon emission computed tomography (SPECT) using $\mathrm{N}$-isopropyl-p-[ $\left.{ }^{123} \mathrm{I}\right]$-iodoamphetamine ${ }^{123} \mathrm{I}$-IMP), which is used for diagnosing uveal melanoma [6], was negative.

Local resection of the tumor was performed under general anesthesia on August 13, 2009. During surgery, the tumor did not transilluminate. First, the pupil was dilated with mydriatic eye drop and the lens was removed by phacoemulsification, and 3 ports were prepared for vitrectomy. After the bulbar conjunctiva 
Ocular Oncology

and Pathology

\begin{tabular}{l|l}
\hline Ocul Oncol Pathol 2015;1:88-92 \\
\hline DOI: $10.1159 / 000369330$ & $\begin{array}{l}\text { ( ) 2014 S. Karger AG, Basel } \\
\text { www.karger.com/oop }\end{array}$ \\
\hline
\end{tabular}

Goto et al.: Perivascular Epithelioid Cell Tumor Arising from Ciliary Body Treated by Local Resection
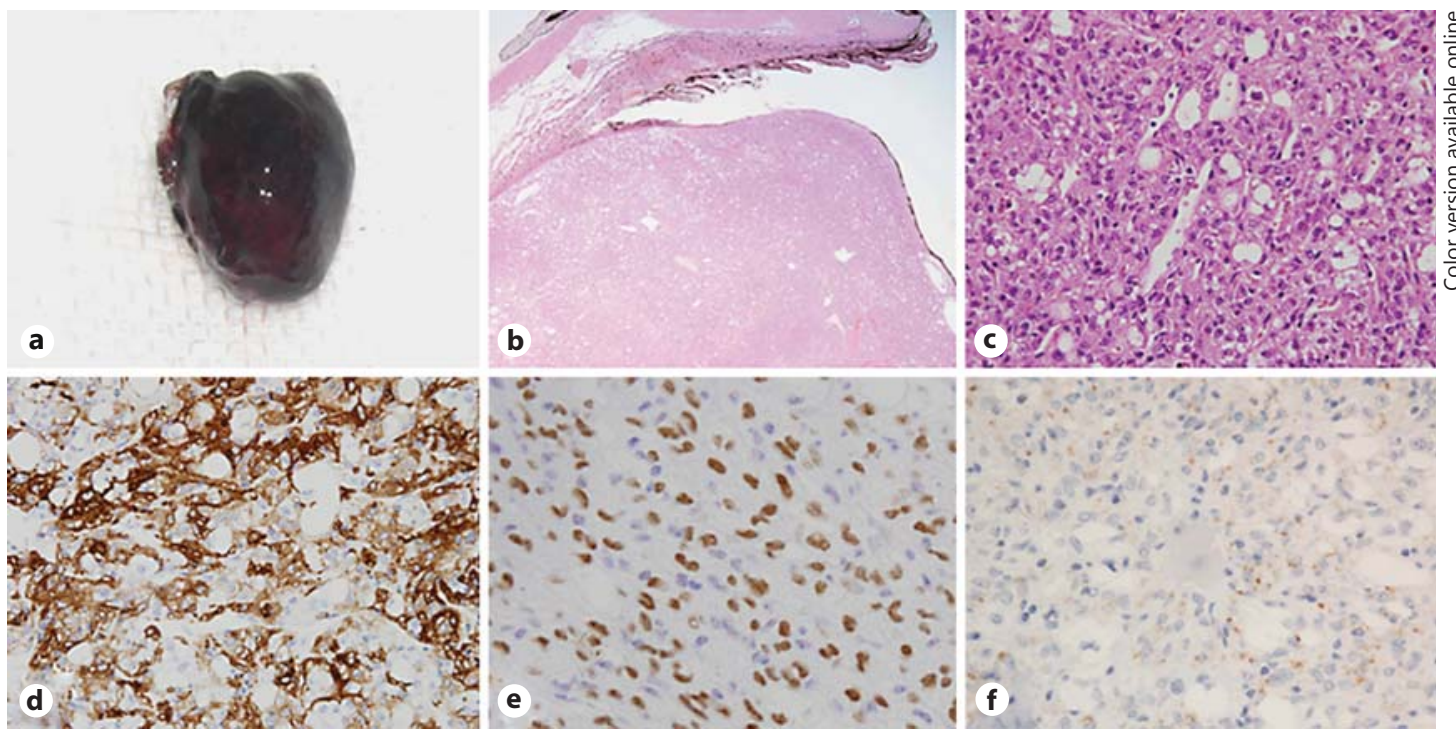

Fig. 2. Histopathology of the excised tumor. a Gross photograph of the resected tumor. $\mathbf{b}$ The tumor arose from the pars plana of the ciliary body (hematoxylin-eosin, original magnification $\times 100$ ). $\mathbf{c}$ Note the proliferated polygonal epithelioid cells with eosinophilic cytoplasm and vacuolated cells (hematoxylin-eosin, $\times 400$ ). d Immunohistochemical staining showed a strong positive reaction for SMA $(\times 400)$. e TFE-3 was diffusely positive in individual cells $(\times 400)$. $\mathbf{f}$ Adipophilin was positive around vacuolated cells $(\times 400)$.

was dissected, a half-thickness scleral flap was made from 6:30 to 11:30 o'clock. The scleral flap was designed from the limbus extending posteriorly, hinged at $8 \mathrm{~mm}$ from the limbus. Cryopexy and diathermy were applied external to the scleral flap to prevent retinal detachment. The base of the sclera and the uvea containing the tumor were incised and subsequently removed together with the iris that was radially dissected in advance. After the scleral flap was replaced and sutured, vitrectomy was performed to remove as much of the vitreous and intraocular hemorrhage as possible. The operation was completed after sulfur hexafluoride gas tamponade.

The excised tumor was brown and $10 \times 11 \times 11 \mathrm{~mm}$ in size (fig. 2a). Histopathologically, the tumor was connected to the ciliary epithelium (fig. $2 \mathrm{~b}$ ) and composed of polygonal and epithelioid cells with clear or eosinophilic cytoplasm (fig. 2c). Foci of tumor cells were lined by fibrovascular tissue. No significant cytological atypia and mitotic figures were noted. Immunohistochemically, the tumor cells were weekly positive for human melanoma black (HMB)-45 and Melan-A in a confined area, and strongly positive for $\alpha$-smooth muscle actin (SMA) (fig. 2d). Transcription factor E (TFE)-3 was diffusely positive (fig. 2e). In addition, adipophilin-positive cells were also observed (fig. 2f). Periodic acid-Schiff stain, desmin, S-100 protein, neuronspecific enolase and glial fibrillary acidic protein were negative. These histopathological and immunohistochemical findings were compatible with the diagnosis of PEComa.

One month after the operation, the patient's best corrected visual acuity was improved to 20/20 OD. No local recurrence of the tumor was observed for more than 4 years after the surgery (fig. 3a, b).

\section{Discussion}

Because of their rarity and variability, it is not easy to make an accurate clinical diagnosis of tumors arising from the ciliary body. Malignant melanoma should be placed at the top of a differential diagnosis, even though the frequency is low in the non-Caucasian population. ${ }^{123}$ I-IMP SPECT is used to make a diagnosis of uveal melanoma [6] and has been reported to be a sensitive and accurate diagnostic tool, far more superior than ${ }^{18} \mathrm{~F}$-FDG PET, for detecting 
Ocular Oncology

and Pathology

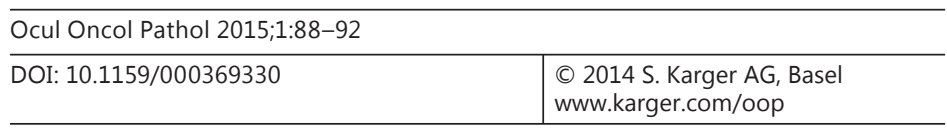

Goto et al.: Perivascular Epithelioid Cell Tumor Arising from Ciliary Body Treated by Local Resection

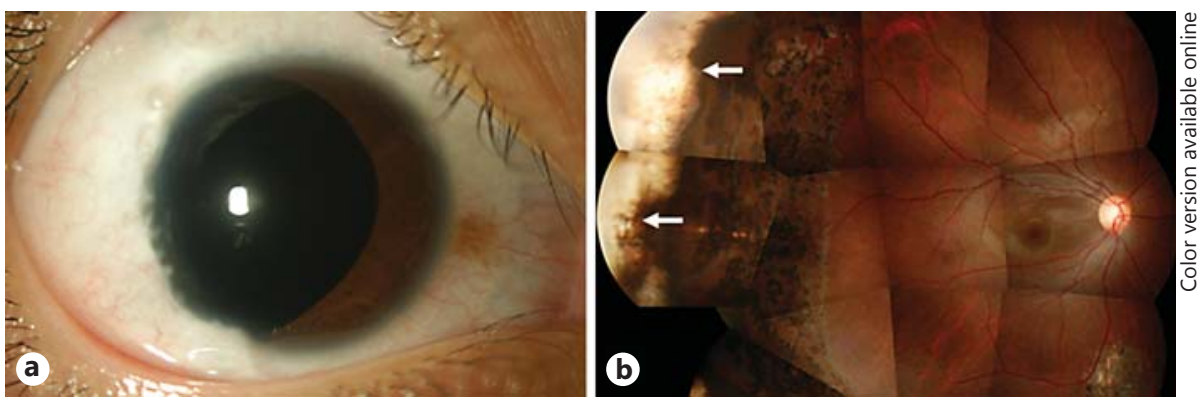

Fig. 3. Slit-lamp and fundus photograph 4 years after local resection of the tumor. a Note the coloboma iridis. b Bare sclera in the peripheral fundus (arrows). Visual acuity was 20/20.

uveal melanoma [7]. In the present case, the result of ${ }^{123}$ I-IMP SPECT was negative, excluding a diagnosis of uveal melanoma.

The results of immunohistochemical staining of the resected tumor were compatible with previous reports of PEComa. According to the World Health Organization classification of tumors, PEComa is defined as 'a mesenchymal tumor composed of histologically and immunohistochemically distinctive perivascular epithelioid cells' [8]. Folpe et al. [9] evaluated 26 PEComas of soft tissue and gynecologic origin and observed expression of at least one melanocytic marker in all cases. HMB-45 was the most sensitive (92\%) followed by Melan-A (72\%), while SMA and desmin were expressed in 80 and 36\%, respectively. They also reported positive expression of TFE-3 in 5 of 17 cases. In the present case, adipophilin was expressed in the tumor. Adipophilin is a protein present in a wide range of cells and tissues, in association with the surface of intracellular lipid droplets [10]. Further research is needed to investigate the significance of adipophilin in PEComa.

Only 4 cases of PEComa with ocular involvement have been reported in the literature. Two of the 4 cases originated from the orbit and 1 from the eyelid [3-5]. The remaining case, a 7-year-old boy who underwent biopsy first and iridocyclectomy 1 year later, was the only case of PEComa identified as intraocular tumor [5]. This young boy was first diagnosed with low-grade melanoma of the balloon-cell type. However, histopathological examinations including immunohistochemistry performed later revealed a positive reaction for HMB-45, SMA and TFE-3. These findings were the same as in the present case and compatible with the diagnosis of PEComa.

The present case was a young girl who had a mature cataract in her right eye. Although a detailed ophthalmic history of our patient is not available, it is possible that the tumor of the ciliary body may have developed over many years and caused the cataract as well as exotropia.

Surgical excision is a challenging method for the treatment of ciliary body tumor. While many studies have evaluated this technique in adults, data on the outcome in pediatric patients are limited [11]. Despite the presence of vitreous hemorrhage for 1 month after surgery, the final best corrected visual acuity in the present case was recovered to $20 / 20$. No recurrence of the tumor and ocular complications was observed for over 5 years, but further follow-up is required.

\section{Disclosure Statement}

The authors declare that there are no conflicts of interest and no financial interests. 
Goto et al.: Perivascular Epithelioid Cell Tumor Arising from Ciliary Body Treated by Local Resection

\section{References}

1 Bonetti F, Pea M, Martignoni G, Zamboni G: PEC and sugar. Am J Surg Pathol 1992;16:307-308.

2 Hornick JL, Fletcher CD: PEComa: what do we know so far? Histopathology 2006;48:75-82.

-3 Iyenqar P, Deangelis DD, Greenberg M, Taylor G: Perivascular epithelioid cell tumor of the orbit: a case report and review of the literature. Pediatr Dev Pathol 2005;8:98-104.

4 Guthoff R, Guthoff T, Mueller-Hermelink HK, et al: Perivascular epithelioid cell tumor of the orbit. Arch Ophthalmol 2008;126:1009-1013.

5 Furusato E, Cameron JD, Newsom RW, et al: Ocular perivascular epithelioid cell tumor: report of 2 cases with distinct clinical presentations. Hum Pathol 2010;41:768-772.

6 Goto H, Usui M, Ishii I: Efficacy of (123)N-isopropyl-p-[(123)I]-iodoamphetamine single photon emission computed tomography for the diagnosis of uveal malignant melanoma. Am J Ophthalmol 2001;132:937-939.

7 Kato K, Kubota T, Ikeda M, et al: Low efficacy of 18F-FDG PET for detection of uveal malignant melanoma compared with 123I-IMP SPECT. J Nucl Med 2006;47:404-409.

8 Folpe AL: Neoplasms with perivascular epithelioid cell differentiation (PEComas); in Fletcher CDM, Unni KK, Epstein J, Mertens F (eds): WHO Classification of Tumours. Pathology and Genetics of Tumours of Soft Tissue and Bone. Lyon, IARC Press, 2002, pp 221-222.

-9 Folpe AL, Mentzel T, Lehr HA, et al: Perivascular epithelioid cell neoplasms of soft tissue and gynecologic origin: a clinicopathologic study of 26 cases and review of the literature. Am J Surg Pathol 2005;29:15581575.

10 Heid HW, Moll R, Schwetlick I, et al: Adipophilin is a specific marker of lipid accumulation in diverse cell types and diseases. Cell Tissue Res 1998;294:309-321.

11 Ramasubramanian A, Shields CL, Kytasty C, Mahmood Z, et al: Resection of intraocular tumors (partial lamellar sclerouvectomy) in the pediatric age group. Ophthalmology 2012;119:2507-2513. 